\title{
Stochastic Nonlinear Thermoelastic System Coupled Sine-Gordon Equation Driven by Jump Noise
}

\author{
Shuilin Cheng, ${ }^{1}$ Yantao Guo, ${ }^{1,2}$ and Yanbin Tang ${ }^{1}$ \\ ${ }^{1}$ School of Mathematics and Statistics, Huazhong University of Science and Technology, Wuhan, Hubei 430074, China \\ ${ }^{2}$ School of Mathematics and Statistics, Xuchang University, Xuchang, Henan 46100, China \\ Correspondence should be addressed to Yanbin Tang; tangybhust@sina.com
}

Received 23 November 2013; Accepted 13 January 2014; Published 24 February 2014

Academic Editor: Ming Mei

Copyright (c) 2014 Shuilin Cheng et al. This is an open access article distributed under the Creative Commons Attribution License, which permits unrestricted use, distribution, and reproduction in any medium, provided the original work is properly cited.

\begin{abstract}
This paper considers a stochastic nonlinear thermoelastic system coupled sine-Gordon equation driven by jump noise. We first prove the existence and uniqueness of strong probabilistic solution of an initial-boundary value problem with homogeneous Dirichlet boundary conditions. Then we give an asymptotic behavior of the solution.
\end{abstract}

\section{Introduction}

In this paper, we consider the following stochastic nonlinear thermoelastic coupled sine-Gordon system driven by Lévy noise:

$$
\begin{gathered}
d u_{t}-\left(\alpha_{1} u_{x x t}+u_{x x}+\beta \sin u+\alpha_{2} \theta\right) d t \\
=\int_{Z} \sigma_{1}(t, u(t), z) \widetilde{\eta_{1}}(d z, d t), \\
d \theta-\left(\theta_{x x}-\alpha_{2} u_{t}+g(t, \theta)\right) d t=\int_{Z} \sigma_{2}(t, \theta(t), z) \widetilde{\eta_{2}}(d z, d t), \\
u(x, t)=0, \quad \theta(x, t)=0, \quad x=0, \quad x=L, t \geq 0, \\
u(x, 0)=u_{0}(x), \quad u_{t}(x, 0)=u_{1}(x), \\
\theta(x, 0)=\theta_{0}(x), \quad x \in[0, L],
\end{gathered}
$$

where $u_{t}=\partial u / \partial t, \tilde{\eta}_{i}(i=1,2)$ are the Lévy processes defined on a complete probability space $(\Omega, \mathscr{F}, P$ ) (see Section 2 for the precise definition) [1-4], $g$ and $\sigma_{i}(i=1,2)$ are given realvalued random functions that will be defined in later.

Recently, the study of high-temperature apparatus or heat resistant structures is becoming important and it is necessary to analyze not only the deterministic thermal stress but also the stochastic thermal stress. In high-temperature apparatus, it is very difficult to predict accurately the thermal environment and mechanical load on its components. Furthermore, many indeterminate factors must be considered, for example, the random high-cycle vibrations of the temperature of the upper core in fast breeder reactors and fluctuations in the heat transfer coefficients around the stationary blades of gas turbines. Therefore, the stochastic case of temperature and thermal stress is indispensable in considering these indeterminate factors of the thermal environment (see [5]).

All the time, a description of wave propagation phenomena in random media is usually based on the study of stochastically perturbed wave equations (see $[6,7])$. In fact, lots of wave phenomena are temperature dependent or heat generating; then the wave equations are coupled with a stochastic heat equation. Caraballo et al. [8] studied the existence of invariant manifolds for coupled parabolic and hyperbolic stochastic partial differential equations. Bates et al. [9] proved the existence of random attractors for stochastic reaction-diffusion equations on unbounded domains, and Wang and Tang $[10,11]$ described the properties of the random attractors.

Meanwhile the sine-Gordon equation is an important model in physics; Fan [12] considered the random attractor for the stochastic sine-Gordon equation. What are the other properties of stochastic sine-Gordon equation? As we know, Coayla-Teran [13] and Liu et al. [3] studied the mild solution 
of stochastic fractional partial differential equation with fractional and jump noises and considered the strong probability solution driven by white or Lévy noise for the stochastic nonlinear nonlocal parabolic equation and 2D stochastic $\mathrm{N}$ $S$ equation. In deterministic coupled case, the well-posedness of the solution for the nonlinear thermoelastic coupled sineGordon system has been studied by many authors, and the global attractor was treated in [14]. Moreover, the more general thermoelastic system coupled model was considered. Gao and Muñoz Rivera [15] and Rivera [16] studied the wellposedness and energy decay rates. In deterministic case, nonlinear thermoelastic system coupled sine-Gordon equation is very weak coupling thermoelastic system; the more general model was investigated by Gao [17]; he considered the global attractor for the semilinear thermoelastic problem.

However, as far as we know, no one refers to the strong solution for stochastic nonlinear thermoelastic coupled sineGordon system by jump noise.

This paper is organized as follows. In the next section, we recall some fundamental results related to the solution of the stochastic equation and Lévy noise. In Section 3, we use the Galerkin method to prove the existence and uniqueness of solution to the problem (1). In Section 4, we give an asymptotic behavior of the solution of the problem (1).

In this paper, $C$ is a constant from line to line.

\section{Preliminaries}

In this section, we recall some fundamental results related to some basic function spaces and the property of Lévy process; for more information, one can see $[1,2,9,12]$. Let $A=$ $-\partial_{x x}$, with the domain $D(A)=H^{2}(0, L) \cap H_{0}^{1}(0, L)$, and $L^{2}(0, L), H_{0}^{1}(0, L)$ are separable Hilbert spaces with the norm $\|\cdot\|$ and $\|\cdot\|_{1}$, respectively; from Poincaré's inequality, $\|\nabla \cdot\|$ is equivalent to $\|\cdot\|_{1}$. Next, we recall some basic concepts related to Lévy process. The readers are referred to [1] for more details.

Let $(Z, \mathscr{Z})$ be a measurable space, and let $\nu$ be a $\sigma$-finite positive measure on it. If $X$ is a topological space, then by $\mathscr{B}(X)$ we will denote the Borel $\sigma$-field on $X$, and $\lambda$ is a Lebesgue measure on $(R, \mathscr{B}(R))$. Suppose that $(\Omega, \mathscr{F}, F, P)$ is a filtered probability space, where $F=\left(\mathscr{F}_{t}\right)_{t \geq 0}$ is a filtration and $\eta_{i}: \Omega \times \mathscr{B}\left(R^{+}\right) \times \mathscr{Z} \rightarrow \bar{N}$ is a time homogeneous Poisson random measure with the intensity measure $v$ defined over the filtered probability space $(\Omega, \mathscr{F}, F, P)$.

We will denote by $\widetilde{\eta}_{i}=\eta_{i}-\gamma_{i}$ the compensated Poisson random measure associated with $\eta_{i}$, where the compensator $\gamma_{i}$ is given by

$$
\mathscr{B}\left(R^{+}\right) \times \mathscr{Z} \ni(A, I) \longmapsto \gamma_{i}(A, I)=\nu(A) \lambda(I) \in R^{+} .
$$

We assume that $\left(H,|\cdot|_{H}\right)$ is a Hilbert space. It is then known (see, for example, $[1,2]$ ) that there exists a unique continuous linear operator $\mathscr{I}$ which associates with each progressively measurable process $\xi: R_{+} \times Z \times \Omega \rightarrow H$ satisfying

$$
E \int_{0}^{T} \int_{Z}|\xi(r, z)|_{H}^{2} \nu(d z) d r<\infty, \quad T>0
$$

Moreover, $\mathscr{I}(\xi)$ is an $H$-valued adapted and càdlàg process such that for any random step process $\xi(r, z)$ satisfying the condition (3) with a representation

$$
\xi(r, z)=\sum_{j=1}^{n} 1_{\left(t_{j-1}, t_{j}\right]}(r) \xi_{j}(z), \quad r \geq 0
$$

where $\left\{0=t_{0}<t_{1}<\cdots<t_{n}<\infty\right\}$ is a partition of $[0, \infty)$, and for all $j, \xi_{j}$ being an $\mathscr{F}_{t_{j-1}}$ measurable random variable, one has

$$
\mathscr{I}(\xi)(t)=\sum_{j=1}^{n} \int_{Z} \xi_{j}(z) \tilde{\eta}\left(d z,\left(t_{j-1} \wedge t, t_{j} \wedge t\right]\right), \quad t \geq 0
$$

In general case we write

$$
\int_{0}^{t} \int_{Z} \xi(r, z) \tilde{\eta}(d z, d r):=\mathscr{I}(\xi)(t), \quad t \geq 0 .
$$

The continuity (more precisely, isometry in Hilbert spaces) of the operator $\mathscr{I}$ mentioned above means that

$$
E\left|\int_{0}^{t} \int_{Z} \xi(r, z) \tilde{\eta}(d z, d r)\right|_{H}^{2}=E \int_{0}^{t} \int_{Z}|\xi(r, z)|_{H}^{2} \nu(d z) d r
$$

The class of all progressively measurable processes $\xi: R_{+} \times$ $Z \times \Omega \rightarrow H$ satisfying the condition (3) will be denoted by $\mathscr{M}^{2}\left(R_{+}, L^{2}(Z, v, H)\right)$. If $T>0$, the class of all progressively measurable processes $\xi:[0, T] \times Z \times \Omega \rightarrow H$ satisfies the condition (3) just for this one $T$, which will be denoted by $\mathscr{M}^{2}\left(0, T, L^{2}(Z, v, H)\right)$.

The main technical tool in our paper is the Itô formula. Let us consider the Hilbert spaces $V \subseteq H \cong H^{\prime} \subseteq V^{\prime}$ and a $V^{\prime}$-valued càdlàg process of the form

$$
\begin{array}{r}
X(t)=X_{0}+\int_{0}^{t} Y(s) d s+\int_{0}^{t} \int_{Z} G(s, z) \widetilde{\eta}(d z, d s), \\
t \in[0, T]
\end{array}
$$

where $Y$ is a $V^{\prime}$-valued process and $G$ is an $H$-valued process; we have the following.

Theorem 1 (see $[1,2])$. Suppose that $X_{0} \in L^{2}\left(\Omega, \mathscr{F} ; V^{\prime}\right)$ and $G \in L^{2}\left(\Omega \times[0, T] ; L^{2}(Z, v ; H)\right)$ are progressively measurable processes. Suppose that $X$ is a $V^{\prime}$-valued process given by (8) and there exists $a V$-valued process $\bar{X} \in L^{2}(\Omega \times[0, T] ; V)$ such that $\bar{X}=X, d P \otimes d t$ in $V$. Then $X$ is an $H$-valued càdlàg $\mathscr{F}_{t^{-}}$ adapted process (up to distinguishable) and

$$
\begin{aligned}
\|X(t)\|^{2}= & \left\|X_{0}\right\|^{2}+2 \int_{0}^{t}\langle\bar{X}, Y(s)\rangle d s \\
& +2 \int_{0}^{t} \int_{Z}(X(s-), G(s, z)) \widetilde{\eta}(d z, d s) \\
& +\int_{0}^{t} \int_{Z}\|G(s, z)\|^{2} \eta(d z, d s) .
\end{aligned}
$$




\section{Existence and Uniqueness of Solution}

In this section, we use the Galerkin method to prove the local existence and uniqueness of solution; then making use of a priori estimates, we prove that there exists a convergence subsequence such that the solution is global.

As is well known, system (1) is equivalent to the following Itô system:

$$
\begin{gathered}
d u=v d t, \\
d v=\left(\alpha_{1} v_{x x}+u_{x x}+\beta \sin u+\alpha_{2} \theta\right) d t \\
+\int_{Z} \sigma_{1}(t, u(t), z) \widetilde{\eta_{1}}(d z, d t), \\
d \theta=\left(\theta_{x x}-\alpha_{2} v+g(t, \theta)\right) d t \\
+\int_{Z} \sigma_{2}(t, \theta(t), z) \widetilde{\eta_{2}}(d z, d t), \\
u(x, t)=0, \quad \theta(x, t)=0, \\
x=0, \quad x=L, \quad t \geq 0, \\
u(x, 0)=u_{0}(x), \quad v(x, 0)=u_{1}(x), \\
\theta(x, 0)=\theta_{0}(x), \quad x \in[0, L] .
\end{gathered}
$$

For simplicity, denote that $g(\theta):=g(t, \theta), \sigma_{i}(u):=$ $\sigma_{i}(t, u, z)(i=1,2)$, and $L^{2}(0, L)=L^{2}, H^{k}(0, L)=H^{k}, k \in$ $N^{+}$. To obtain the existence of solution to (10), we suppose that the functions

$$
\begin{gathered}
\sigma_{i}:[0, \infty) \times L^{2}(0, L) \longrightarrow L^{2}\left(Z, v ; L^{2}(0, L)\right), \\
g:[0, \infty) \times L^{2}(0, L) \longrightarrow L^{2}(0, L)
\end{gathered}
$$

satisfy the following conditions:

$$
\begin{gathered}
\mathrm{C}_{1}:\left\|\sigma_{i}(u)\right\|_{L^{2}\left(Z, v ; L^{2}(0, L)\right)}^{2} \leq k_{1}\|u\|^{2}, \quad\|g(u)\|^{2} \leq K_{1}\|u\|^{2}, \\
\mathrm{C}_{2}:\left\|\sigma_{i}(u)-\sigma_{i}(v)\right\|_{L^{2}\left(Z, v ; L^{2}(0, L)\right)}^{2} \leq k_{2}\|u-v\|^{2}, \\
\|g(u)-g(v)\|^{2} \leq K_{2}\|u-v\|^{2},
\end{gathered}
$$

where $k_{i}, K_{i}>0, i=1,2$ and $u, v \in L^{2}(0, L)$.

Definition 2. An $\mathscr{F}_{t}$-adapted stochastic process $\{(u(t), v(t)$, $\theta(t))\}_{t \geq 0}$ is said to be a strong probabilistic solution of stochastic nonlinear thermoelastic coupled system driven by Lévy noise (10) if it satisfies the following:

(1) $(u(t), v(t), \theta(t)) \in L^{2}\left(\Omega ; C\left([0, T] ; H^{1} \times L^{2} \times L^{2}\right)\right)$ a.s. for any $T>0$;

(2) the identities

$$
\left(u(t), \varphi_{1}\right)=\left(u_{0}, \varphi_{1}\right)+\int_{0}^{t}\left(v(s), \varphi_{1}\right) d s,
$$

$$
\begin{aligned}
\left(v(t), \varphi_{2}\right)= & \left(v_{0}, \varphi_{2}\right) \\
& +\int_{0}^{t}\left(\alpha_{1} v_{x x}+u_{x x}+\beta \sin u+\alpha_{2} \theta, \varphi_{2}\right) d s \\
& +\int_{0}^{t} \int_{Z}\left(\sigma_{1}(s, u(s), z), \varphi_{2}\right) \widetilde{\eta_{1}}(d z, d s), \\
\left(\theta(t), \varphi_{3}\right)= & \left(\theta_{0}, \varphi_{3}\right)+\int_{0}^{t}\left(\theta_{x x}-\alpha_{2} v+g(\theta), \varphi_{3}\right) d s \\
& +\int_{0}^{t} \int_{Z}\left(\sigma_{2}(s, \theta(s), z), \varphi_{3}\right) \widetilde{\eta_{2}}(d z, d s),
\end{aligned}
$$

hold $P$-a.s. for all $\left(\varphi_{1}, \varphi_{2}, \varphi_{3}\right) \in L^{2} \times H_{0}^{1} \times H_{0}^{1}$.

We now give our main result.

Theorem 3. Assume that $\left(u_{0}, v_{0}, \theta_{0}\right) \in H^{1} \times L^{2} \times L^{2}$ is $\mathscr{F}_{0^{-}}$ adapted. The conditions $C_{1}$ and $C_{2}$ are satisfied. $\widetilde{\eta_{i}}=\eta_{i}-\gamma_{i}(i=$ $1,2)$ are the compensated Poisson random measure associated with $\eta_{i}$, where the compensator $\gamma_{i}$ is defined in Section 2. Then for any $T>0$ the stochastic nonlinear thermoelastic coupled system driven by Lévy noise (10) has a unique solution

$$
(u(t), v(t), \theta(t)) \in L^{2}\left(\Omega ; C\left([0, T] ; H^{1} \times L^{2} \times L^{2}\right)\right)
$$

such that

$$
E \sup _{0 \leq t \leq T}\left(\|u(t)\|_{1}^{2}+\|v(t)\|^{2}+\|\theta(t)\|^{2}\right) \leq C,
$$

where $C$ is a positive constant.

Proof. Existence. We use the Galerkin approximation and some useful a priori estimates to prove the existence of solution. Set

$$
\begin{gathered}
u_{n}(t)=\sum_{i=1}^{n} a_{i} \phi_{i}, \quad v_{n}(t)=\sum_{i=1}^{n} b_{i} \phi_{i}, \\
\theta_{n}(t)=\sum_{i=1}^{n} c_{i} \phi_{i},
\end{gathered}
$$

where $\left\{\phi_{i}\right\}_{i=1}^{\infty}$ is the set of eigenfunctions of $-\partial_{x x}$ with domain $\left.H^{2}(0, L) \cap H_{0}^{1}(0, L)\right)$; it is an orthogonal set of $H=L^{2}$ and orthonormal one in $H_{0}^{1}, a_{i}=\left(u, \phi_{i}\right), b_{i}=\left(v, \phi_{i}\right)$, and $c_{i}=$ $\left(\theta, \phi_{i}\right)$. Denote by $P_{n}: H \rightarrow H_{n}$ the orthogonal projector, where $H_{n}=\operatorname{Span}\left\{\phi_{1}, \ldots, \phi_{n}\right\}$.

Hence, we can rewrite (10) as

$$
\begin{gathered}
d u_{n}=v_{n} d t, \\
d v_{n}=\left(\alpha_{1} v_{n x x}+u_{n x x}+\beta P_{n} \sin u_{n}+\alpha_{2} \theta_{n}\right) d t \\
+\int_{Z} P_{n} \sigma_{1}\left(u_{n}\right) \widetilde{\eta_{1}}(d z, d t), \\
d \theta_{n}=\left(\theta_{n x x}-\alpha_{2} v_{n}+P_{n} g\left(\theta_{n}\right)\right) d t \\
+\int_{Z} P_{n} \sigma_{2}\left(\theta_{n}\right) \widetilde{\eta_{2}}(d z, d t),
\end{gathered}
$$




$$
\begin{gathered}
u_{n}(x, t)=0, \quad \theta_{n}(x, t)=0, \quad x=0, \quad x=L, t \geq 0, \\
u_{n}(x, 0)=u_{0 n}(x), \\
v_{n}(x, 0)=u_{1 n}(x)=v_{0 n}(x), \\
\theta_{n}(x, 0)=\theta_{0 n}(x),
\end{gathered}
$$$$
x \in[0, L],
$$

where, for each $n \in N^{+},\left(u_{0 n}, v_{0 n}, \theta_{0 n}\right) \rightarrow\left(u_{0}, v_{0}, \theta_{0}\right)$ in $H^{1} \times$ $L^{2} \times L^{2}$.

Applying Itô formula to the process $\left\|v_{n}(t)\right\|^{2}$, we obtain

$$
\begin{aligned}
\left\|v_{n}(t)\right\|^{2}= & \left\|v_{0 n}\right\|^{2}+\int_{0}^{t} \int_{Z}\left\|P_{n} \sigma_{1}\left(u_{n}\right)\right\|^{2} \eta_{1}(d z, d s) \\
& +2 \int_{0}^{t} \int_{Z}\left(v_{n}(s-), P_{n} \sigma_{1}\left(u_{n}\right)\right) \widetilde{\eta_{1}}(d z, d s) \\
& +2 \int_{0}^{t}\left(v_{n}, \alpha_{1} v_{n x x}+u_{n x x}+\beta P_{n} \sin u_{n}+\alpha_{2} \theta_{n}\right) d s \\
= & \left\|v_{0 n}\right\|^{2}+\left\|u_{0 n}\right\|_{1}^{2}-\left\|u_{n}(t)\right\|_{1}^{2} \\
& -2 \alpha_{1} \int_{0}^{t}\left\|v_{n}(t)\right\|_{1}^{2} d s \\
& +2 \int_{0}^{t}\left(v_{n}, \beta P_{n} \sin u_{n}+\alpha_{2} \theta_{n}\right) d s \\
& +2 \int_{0}^{t} \int_{Z}^{t}\left(v_{n}(s-), P_{n} \sigma_{1}\left(u_{n}\right)\right) \widetilde{\eta_{1}}(d z, d s) \\
& +\int_{0}^{t} \int_{Z}\left\|P_{n} \sigma_{1}\left(u_{n}\right)\right\|^{2} \eta_{1}(d z, d s) .
\end{aligned}
$$

Denote that

$$
E(t):=\int_{0}^{t} \int_{Z}\left(u_{n}(s-), P_{n} \sigma_{1}\left(u_{n}\right)\right) \widetilde{\eta_{1}}(d z, d s) .
$$

Since $\left\{u_{n}(t)\right\}_{t \in[0, T]}$ is an adapted and càdlàg process, the process $E(t)$ is a martingale. Applying the Burkholder-DavisGundy inequality, condition $\mathrm{C}_{1}$, Hölder's inequality, and Young's inequality, we get

$$
\begin{aligned}
& E \sup _{0 \leq s \leq t} \int_{0}^{s} \int_{Z}\left(v_{n}(r-), P_{n} \sigma_{1}\left(u_{n}\right)\right) \widetilde{\eta_{1}}(d z, d r) \\
& \leq 3 E\left[\int_{0}^{t} \int_{Z}\left\|v_{n}(s-)\right\|^{2}\left\|P_{n} \sigma_{1}\left(u_{n}\right)\right\|^{2} v(d z) d s\right]^{1 / 2} \\
& \leq 3\left[\sup _{0 \leq s \leq t}\left\|v_{n}(s)\right\|^{2}\right]^{1 / 2}\left[k_{1} \int_{0}^{t}\left\|u_{n}(s)\right\|^{2} d s\right]^{1 / 2} \\
& \leq \frac{1}{4} E \sup _{0 \leq s \leq t}\left\|v_{n}(s)\right\|^{2}+9 k_{1} E \int_{0}^{t}\left\|u_{n}(s)\right\|^{2} d s .
\end{aligned}
$$

Taking into account that the process

$$
t \longmapsto \int_{0}^{t} \int_{Z}\left\|P_{n} \sigma_{1}\left(u_{n}\right)\right\|^{2} \eta_{1}(d z, d s)
$$

has only positive jumps, we obtain

$$
\begin{aligned}
& E \sup _{0 \leq s \leq t} \int_{0}^{s} \int_{Z}\left\|P_{n} \sigma_{1}\left(u_{n}\right)\right\|^{2} \eta_{1}(d z, d r) \\
& \quad \leq E \int_{0}^{t} \int_{Z}\left\|\sigma_{1}\left(u_{n}\right)\right\|^{2} v(d z) d s \\
& \quad \leq k_{1} E \int_{0}^{t}\left\|u_{n}(s)\right\|^{2} d s .
\end{aligned}
$$

From the Hölder inequality, we have

$$
\left|\int_{0}^{t}\left(v_{n}, \beta P_{n} \sin u_{n}\right) d s\right| \leq \int_{0}^{t}\left\|v_{n}(s)\right\|^{2} d s+C t .
$$

Putting (20)-(23) into (18), for $t \in[0, T]$, we have

$$
\begin{aligned}
E \sup _{0 \leq s \leq t}\left(\left\|u_{n}\right\|_{1}^{2}+\frac{1}{2}\left\|v_{n}\right\|^{2}\right) & \\
\leq & \left\|u_{0 n}\right\|_{1}^{2}+\left\|v_{0 n}\right\|^{2}-2 \alpha_{1} E \int_{0}^{t}\left\|v_{n}\right\|_{1}^{2} d s \\
& +2 E \int_{0}^{t}\left\|v_{n}\right\|^{2} d s+19 k_{1} E \int_{0}^{t}\left\|u_{n}(s)\right\|^{2} d s \\
& +2 \alpha_{2} E \int_{0}^{t}\left(v_{n}, \theta_{n}\right) d s+C t .
\end{aligned}
$$

Similarly, using the Itô formula to the process $\left\|\theta_{n}(t)\right\|^{2}$, we obtain

$$
\begin{aligned}
\left\|\theta_{n}(t)\right\|^{2}= & \left\|\theta_{0 n}\right\|^{2}+2 \int_{0}^{t}\left(\theta_{n}, \theta_{n x x}-\alpha_{2} v_{n}+P_{n} g\left(\theta_{n}\right)\right) d s \\
& +\int_{0}^{t} \int_{Z}\left\|P_{n} \sigma_{2}\left(\theta_{n}(s)\right)\right\|^{2} \eta_{2}(d z, d s) \\
& +2 \int_{0}^{t} \int_{Z}\left(\theta_{n}(s-), P_{n} \sigma_{2}\left(\theta_{n}(s)\right)\right) \widetilde{\eta}_{2}(d z, d s) .
\end{aligned}
$$

Due to the Burkholder-Davis-Gundy inequality, condition $\mathrm{C}_{1}$, the Hölder inequality, and the Young inequality,

$$
\begin{gathered}
E \sup _{0 \leq s \leq t} \int_{0}^{s} \int_{Z}\left(\theta_{n}(r-), P_{n} \sigma_{2}\left(\theta_{n}\right)\right) \widetilde{\eta_{2}}(d z, d r) \\
\leq \frac{1}{4} E \sup _{0 \leq s \leq t}\left\|\theta_{n}(s)\right\|^{2}+9 k_{1} E \int_{0}^{t}\left\|\theta_{n}(s)\right\|^{2} d s, \\
E \sup _{0 \leq s \leq t} \int_{0}^{s} \int_{Z}\left\|P_{n} \sigma_{2}\left(\theta_{n}\right)\right\|^{2} \eta_{2}(d z, d r) \leq k_{1} E \int_{0}^{t}\left\|\theta_{n}(s)\right\|^{2} d s, \\
2 \int_{0}^{t}\left(\theta_{n}, P_{n} g\left(\theta_{n}\right)\right) d s \leq\left(1+K_{1}\right) \int_{0}^{t}\left\|\theta_{n}\right\|^{2} d s .
\end{gathered}
$$


Hence, we get

$$
\begin{aligned}
\frac{1}{2} E \sup _{0 \leq s \leq t}\left\|\theta_{n}(t)\right\|^{2} \leq & \left\|\theta_{0 n}\right\|^{2}+\left(1+K_{1}\right) E \\
& \times \int_{0}^{t}\left\|\theta_{n}\right\|^{2} d s-2 \alpha_{2} E \int_{0}^{t}\left(v_{n}, \theta_{n}\right) d s \\
& -2 E \int_{0}^{t}\left\|\theta_{n}\right\|^{2} d s+19 k_{1} E \\
& \times \int_{0}^{t}\left\|\theta_{n}(s)\right\|^{2} d s+C t .
\end{aligned}
$$

For every $t \in[0, T],(24)$ and (27) imply that

$$
\begin{aligned}
E \sup _{0 \leq s \leq t}\left(\left\|u_{n}\right\|_{1}^{2}+\frac{1}{2}\left\|v_{n}\right\|^{2}+\frac{1}{2}\left\|\theta_{n}\right\|^{2}\right) \\
\quad+2 \alpha_{1} E \int_{0}^{t}\left\|v_{n}\right\|_{1}^{2} d s+2 E \int_{0}^{t}\left\|\theta_{n}\right\|_{1}^{2} d s \\
\leq\left\|u_{0 n}\right\|_{1}^{2}+\left\|v_{0 n}\right\|^{2}+\left\|\theta_{0 n}\right\|^{2} \\
+\beta E \int_{0}^{t}\left(\left\|u_{n}\right\|_{1}^{2}+\left\|v_{n}\right\|^{2}+\left\|\theta_{n}\right\|^{2}\right) d s+C t,
\end{aligned}
$$

where $\beta=\max \left\{4,2\left(1+K_{1}+19 k_{1}\right)\right\}$.

Then the Gronwall lemma implies that

$$
E \sup _{0 \leq s \leq t}\left(\left\|u_{n}(t)\right\|_{1}^{2}+\left\|v_{n}(t)\right\|^{2}+\left\|\theta_{n}(t)\right\|^{2}\right) \leq C t .
$$

Substituting (29) into (28), we get

$$
E \int_{0}^{t}\left(\left\|v_{n}(s)\right\|_{1}^{2}+\left\|\theta_{n}(s)\right\|_{1}^{2}\right) d s \leq C t
$$

To complete the proof of the existence of solution we need to pass the limits in the Galerkin approximation. Owing to (29) and (30), there exists a subsequence of $\left\{\left(u_{n}, v_{n}, \theta_{n}\right)\right\}$, not relabeled, such that

$$
\begin{gathered}
u_{n} \rightarrow \widehat{u} \text { in } L^{2}\left(\Omega ; C\left([0, t] ; H^{1}(0, L)\right)\right), \\
v_{n} \rightarrow \widehat{v} \text { in } L^{2}\left(\Omega ; C\left([0, t] ; L^{2}(0, L)\right)\right) \\
\cap L^{2}\left(\Omega \times[0, t] ; H^{1}(0, L)\right), \\
\theta_{n} \rightarrow \widehat{\theta} \text { in } L^{2}\left(\Omega ; C\left([0, t] ; L^{2}(0, L)\right)\right) \\
\cap L^{2}\left(\Omega \times[0, t] ; H^{1}(0, L)\right) .
\end{gathered}
$$

From the conditions in $g, \sigma_{i}(i=1,2)$, and (29)-(30), $\forall t \in$ $[0, T]$, we have

$$
\begin{aligned}
& E \int_{0}^{t}\left\|P_{n} \sin \left(u_{n}\right)\right\|^{2} d s<\infty, \\
& E \int_{0}^{t}\left\|P_{n} g\left(s, \theta_{n}\right)\right\|^{2} d s<\infty,
\end{aligned}
$$

$$
\begin{gathered}
E \int_{0}^{t} \int_{Z}\left\|P_{n} \sigma_{1}\left(u_{n}\right)\right\|^{2} v(d z) d s \\
\leq k_{1} E \int_{0}^{t}\left\|u_{n}(s)\right\|^{2} d s<\infty, \\
E \int_{0}^{t} \int_{Z}\left\|P_{n} \sigma_{2}\left(\theta_{n}\right)\right\|^{2} v(d z) d s \\
\quad \leq k_{1} E \int_{0}^{t}\left\|\theta_{n}(s)\right\|^{2} d s<\infty .
\end{gathered}
$$

Hence, there exist the functions

$$
\begin{gathered}
f^{*}, g^{*} \in L^{2}\left(\Omega \times[0, t] ; L^{2}(0, L)\right), \\
\sigma_{i}^{*} \in L^{2}\left(\Omega \times[0, t] ; L^{2}\left(Z, v ; L^{2}(0, L)\right)\right), \quad(i=1,2)
\end{gathered}
$$

such that

$$
\begin{gathered}
P_{n} \sin \left(u_{n}\right) \rightarrow f^{*} \text { in } L^{2}\left(\Omega \times[0, t] ; L^{2}(0, L)\right), \\
P_{n} g\left(\cdot, \theta_{n}\right) \rightarrow g^{*} \text { in } L^{2}\left(\Omega \times[0, t] ; L^{2}(0, L)\right), \\
P_{n} \sigma_{1}\left(\cdot, u_{n}\right) \rightarrow \sigma_{1}^{*} \text { in } L^{2}\left(\Omega \times[0, t] ; L^{2}\left(Z, v ; L^{2}(0, L)\right)\right), \\
P_{n} \sigma_{2}\left(\cdot, \theta_{n}\right) \rightarrow \sigma_{2}^{*} \text { in } L^{2}\left(\Omega \times[0, t] ; L^{2}\left(Z, v ; L^{2}(0, L)\right)\right) .
\end{gathered}
$$

Combining (31) and (34) and letting $n \rightarrow \infty$ in (17), since the linear map

$$
f \longmapsto \int_{0}^{t} \int_{Z} f(s, z) \widetilde{\eta_{i}}(d z, d s)
$$

is continuous from $L^{2}\left(\Omega ; L^{2}\left(Z, v ; L^{2}(0, L)\right)\right)$ to $L^{2}\left(\Omega ; L^{2}(0, L)\right)$ (in fact an isometry), it is continuous with respect to the weak topologies. Therefore, in view of the weak convergence, we have

$$
\left(\widehat{u}(t), \varphi_{1}\right)=\left(u_{0}, \varphi_{1}\right)+\int_{0}^{t}\left(\widehat{v}(s), \varphi_{1}\right) d s,
$$$$
\left(\widehat{v}(t), \varphi_{2}\right)=\left(v_{0}, \varphi_{2}\right)
$$

$$
\begin{aligned}
& +\int_{0}^{t}\left(\alpha_{1} \widehat{v}_{x x}+\widehat{u}_{x x}+\beta f^{*}+\alpha_{2} \widehat{\theta}, \varphi_{2}\right) d s \\
& +\int_{0}^{t} \int_{Z}\left(\sigma_{1}^{*}(s, z), \varphi_{2}\right) \widetilde{\eta_{1}}(d z, d s),
\end{aligned}
$$

$$
\begin{aligned}
\left(\widehat{\theta}(t), \varphi_{3}\right)= & \left(\theta_{0}, \varphi_{3}\right) \\
& +\int_{0}^{t}\left(\widehat{\theta}_{x x}-\alpha_{2} \widehat{v}+g^{*}, \varphi_{3}\right) d s \\
& +\int_{0}^{t} \int_{Z}\left(\sigma_{2}^{*}(s, z), \varphi_{3}\right) \widetilde{\eta}_{2}(d z, d s),
\end{aligned}
$$

for almost everywhere $(\omega, t) \in \Omega \times[0, T]$ and $\left(\varphi_{1}, \varphi_{2}, \varphi_{3}\right) \in$ $L^{2} \times H_{0}^{1} \times H_{0}^{1}$. 
Denote by $\{(u(t), v(t), \theta(t))\}_{t \in[0, T]}$ the process which has a.s. sample paths being continuous in $\left(H^{1} \times L^{2} \times L^{2}\right)$, is $\mathscr{F}_{t}$-adapted, and equals to $\{(\widehat{u}(t), \widehat{v}(t), \widehat{\theta}(t))\}_{t \in[0, T]}$ almost everywhere $(\omega, t) \in \Omega \times[0, T]$; then from (34) we obtain

$$
\begin{aligned}
\left(u(t), \varphi_{1}\right) & =\left(u_{0}, \varphi_{1}\right)+\int_{0}^{t}\left(v(s), \varphi_{1}\right) d s, \\
\left(v(t), \varphi_{2}\right)= & \left(v_{0}, \varphi_{2}\right) \\
+ & \int_{0}^{t}\left(\alpha_{1} v_{x x}+u_{x x}+\beta f^{*}+\alpha_{2} \theta, \varphi_{2}\right) d s \\
& +\int_{0}^{t} \int_{Z}\left(\sigma_{1}^{*}(s, z), \varphi_{2}\right) \widetilde{\eta_{1}}(d z, d s), \\
\left(\theta(t), \varphi_{3}\right)= & \left(\theta_{0}, \varphi_{3}\right) \\
& +\int_{0}^{t}\left(\theta_{x x}-\alpha_{2} v+g^{*}, \varphi_{3}\right) d s \\
& +\int_{0}^{t} \int_{Z}\left(\sigma_{2}^{*}(s, z), \varphi_{3}\right) \widetilde{\eta_{2}}(d z, d s) .
\end{aligned}
$$

Now, we consider the stopping time; for each $N \in N^{+}$,

$$
\tau_{N}=\left\{\begin{array}{c}
\inf \left\{t \in[0, T]:\|u(t)\|_{1}^{2} \vee\|v(t)\|^{2} \vee\|\theta(t)\|^{2}>N\right\}, \\
T,\|u(t)\|_{1}^{2} \vee\|v(t)\|^{2} \vee\|\theta(t)\|^{2} \leq N .
\end{array}\right.
$$

We claim that $\{(u(t), v(t), \theta(t))\}_{t \in[0, T]}$ holds:

$$
\begin{gathered}
\lim _{n \rightarrow \infty} E\left(\left\|\left(u-u_{n}\right)\left(\tau_{N}\right)\right\|_{1}^{2}+\left\|\left(v-v_{n}\right)\left(\tau_{N}\right)\right\|^{2}\right. \\
\left.+\left\|\left(\theta_{n}-\theta_{n}\right)\left(\tau_{N}\right)\right\|^{2}\right)=0, \\
\lim _{n \rightarrow \infty} E \int_{0}^{\tau_{N}}\left[\left\|\left(v-v_{n}\right)(s)\right\|_{1}^{2}+\left\|\left(\theta-\theta_{n}\right)(s)\right\|_{1}^{2}\right] d s=0, \\
\lim _{n \rightarrow \infty} E \int_{0}^{\tau_{N}}\left\|\sigma_{1}^{*}-P_{n} \sigma_{1}(u)\right\|_{L^{2}\left(Z, v ; L^{2}\right)}^{2} d s=0, \\
\lim _{n \rightarrow \infty} E \int_{0}^{\tau_{N}}\left\|\sigma_{2}^{*}-P_{n} \sigma_{2}(\theta)\right\|_{L^{2}\left(Z, v L^{2}\right)}^{2} d s=0 .
\end{gathered}
$$

From (17) and (37), for any $\left(\phi_{i}, \phi_{j}, \phi_{k}\right) \in H_{0}^{1} \times\left(H^{2} \cap H_{0}^{1}\right) \times$ $H_{0}^{1}, i, j, k \in[1, n]$, we have

$$
\begin{gathered}
\left(\left(u-u_{n}\right)(t), \phi_{i}\right)=\int_{0}^{t}\left(\left(v-v_{n}\right)(s), \phi_{i}\right) d s, \\
\left(\left(v-v_{n}\right)(t), \phi_{j}\right) \\
=\int_{0}^{t}\left(\alpha_{1}\left(v-v_{n}\right)_{x x}+\left(u-u_{n}\right)_{x x}\right. \\
\left.\quad+\beta\left(f^{*}-P_{n} \sin u_{n}\right), \phi_{j}\right) d s \\
+\int_{0}^{t}\left(\alpha_{2}\left(\theta-\theta_{n}\right), \phi_{j}\right) d s \\
+\int_{0}^{t} \int_{Z}\left(\sigma_{1}^{*}(s, z)-P_{n} \sigma_{1}\left(u_{n}\right), \phi_{j}\right) \widetilde{\eta_{1}}(d z, d s),
\end{gathered}
$$

$$
\begin{aligned}
& \left(\left(\theta-\theta_{n}\right)(t), \phi_{k}\right) \\
& =\int_{0}^{t}\left(\left(\theta-\theta_{n}\right)_{x x}-\alpha_{2}\left(v-v_{n}\right)\right. \\
& \left.\quad+\left(g^{*}-P_{n} g\left(\theta_{n}\right)\right), \phi_{k}\right) d s \\
& \quad+\int_{0}^{t} \int_{Z}\left(\sigma_{2}^{*}(s, z)-P_{n} \sigma_{2}\left(\theta_{n}\right), \phi_{k}\right) \widetilde{\eta_{2}}(d z, d s),
\end{aligned}
$$

a.s., for all $t \in[0, T]$.

For each $n \in N^{+}$, let

$$
\begin{aligned}
& P_{n}(u(t))=\widetilde{u}_{n}(t)=\sum_{i=1}^{n}\left(u(t), \phi_{i}\right) \phi_{i}, \\
& P_{n}(v(t))=\widetilde{v}_{n}(t)=\sum_{j=1}^{n}\left(v(t), \phi_{j}\right) \phi_{j}, \\
& P_{n}(\theta(t))=\widetilde{\theta}_{n}(t)=\sum_{k=1}^{n}\left(\theta(t), \phi_{k}\right) \phi_{k} .
\end{aligned}
$$

Set $\rho(t)=e^{-\delta t}$, where $\delta$ is a positive constant to be defined later. By applying Itô's formula to the processes $\rho(t)\left\|\widetilde{v}_{n}(t)-v_{n}(t)\right\|^{2}, \quad \rho(t)\left\|\widetilde{u}_{n}(t)-u_{n}(t)\right\|_{1}^{2}$, and $\rho(t)\left\|\widetilde{\theta}_{n}(t)-\theta_{n}(t)\right\|^{2}$, respectively, we obtain

$$
\begin{aligned}
& \rho(t)\left\|\widetilde{v}_{n}(t)-v_{n}(t)\right\|^{2}+\rho(t)\left\|\widetilde{u}_{n}(t)-u_{n}(t)\right\|_{1}^{2} \\
&=-2 \alpha_{1} \int_{0}^{t} \rho(s)\left\|\widetilde{v}_{n}(s)-v_{n}(s)\right\|_{1}^{2} d s \\
&+\int_{0}^{t} \rho^{\prime}(s)\left\|\tilde{u}_{n}(s)-u_{n}(s)\right\|_{1}^{2} d s \\
&+2 \beta \int_{0}^{t} \rho(s)\left(\widetilde{v}_{n}(s)-v_{n}(s),\right. \\
&\left.+2 \alpha_{2} \int_{0}^{t} \rho(s)\left(\tilde{v}_{n}(s)-v_{n}(s), \sin u_{n}(s)\right)\right) d s \\
&+\int_{0}^{t} \rho^{\prime}(s)\left\|\widetilde{v}_{n}(s)-v_{n}(s)\right\|^{2} d s \\
&+2 \int_{0}^{t} \int_{Z} \rho(s)\left(\widetilde{v}_{n}(s-)-v_{n}(s-),\right. \\
&+\int_{0}^{t} \int_{Z} \rho(s)\left\|P_{n} \sigma_{1}^{*}(s, z)-P_{n} \sigma_{1}\left(u_{n}\right)\right\|^{2} \eta_{1}(d z, d s), \\
& \rho(t)\left\|\widetilde{\theta}_{n}(t)-\theta_{n}(t)\right\| \sigma_{1}^{2} \\
&=-2 \int_{0}^{t} \rho(s)\left\|\widetilde{\theta}_{n}(s)-\theta_{n}(s)\right\|_{1}^{2} d s \\
&+\int_{0}^{t} \rho^{\prime}(s)\left\|\widetilde{\theta}_{n}(s)-\theta_{n}(s)\right\|^{2} d s
\end{aligned}
$$




$$
\begin{aligned}
& -2 \alpha_{2} \int_{0}^{t} \rho(s)\left(\widetilde{v}_{n}(s)-v_{n}(s), \widetilde{\theta}_{n}(s)-\theta_{n}(s)\right) d s \\
& +\int_{0}^{t} \int_{Z} \rho(s)\left\|P_{n} \sigma_{2}^{*}(s, z)-P_{n} \sigma_{2}\left(\theta_{n}\right)\right\|^{2} \eta_{2}(d z, d s) \\
& +2 \int_{0}^{t} \rho(s)\left(\widetilde{\theta}_{n}(s)-\theta_{n}(s), P_{n} g^{*}(s)-P_{n} g\left(\theta_{n}\right)\right) d s \\
& +2 \int_{0}^{t} \int_{Z} \rho(s)\left(\widetilde{\theta}_{n}(s-)-\theta_{n}(s-),\right. \\
& \left.P_{n} \sigma_{2}^{*}(s, z)-P_{n} \sigma_{2}\left(\theta_{n}\right)\right)(d z, d s) .
\end{aligned}
$$

Taking the mathematical expectations in (42) yields

$$
\begin{aligned}
& E\left\{\rho ( t ) \left(\left\|\widetilde{v}_{n}(t)-v_{n}(t)\right\|^{2}+\left\|\widetilde{u}_{n}(t)-u_{n}(t)\right\|_{1}^{2}\right.\right. \\
& \left.\left.+\left\|\widetilde{\theta}_{n}(t)-\theta_{n}(t)\right\|^{2}\right)\right\} \\
& +E \int_{0}^{t} \rho(s)\left[2 \alpha_{1}\left\|\widetilde{v}_{n}(s)-v_{n}(s)\right\|_{1}^{2}+\left\|\widetilde{\theta}_{n}(s)-\theta_{n}(s)\right\|_{1}^{2}\right] d s \\
& =E \int_{0}^{t} \rho^{t}(s)\left[\left\|\widetilde{u}_{n}(s)-u_{n}(s)\right\|_{1}^{2}+\left\|\widetilde{v}_{n}(s)-v_{n}(s)\right\|^{2}\right. \\
& \left.\quad+\left\|\widetilde{\theta}_{n}(s)-\theta_{n}(s)\right\|^{2}\right] d s \\
& +2 \beta E \int_{0}^{t} \rho(s)\left(\widetilde{v}_{n}(s)-v_{n}(s), P_{n}\left(f^{*}(s)-\sin u_{n}(s)\right)\right) d s \\
& +E \int_{0}^{t} \int_{Z} \rho(s)\left\|P_{n} \sigma_{1}^{*}(s, z)-P_{n} \sigma_{1}\left(u_{n}\right)\right\|^{2} \eta_{1}(d z, d s) \\
& +E \int_{0}^{t} \int_{Z} \rho(s)\left\|P_{n} \sigma_{2}^{*}(s, z)-P_{n} \sigma_{2}\left(\theta_{n}\right)\right\|^{2} \eta_{2}(d z, d s) \\
& +2 E \int_{0}^{t} \rho(s)\left(\widetilde{\theta}_{n}(s)-\theta_{n}(s), P_{n} g^{*}(s)-P_{n} g\left(\theta_{n}\right)\right) d s .
\end{aligned}
$$

Let us analyze each term of (43). By the conditions $C_{1}$ and $C_{2}$, we have

$$
\begin{aligned}
\left(\widetilde{\theta}_{n}-\right. & \left.\theta_{n}, P_{n} g^{*}-P_{n} g\left(\theta_{n}\right)\right) \\
= & \left(\widetilde{\theta}_{n}-\theta_{n}, P_{n} g^{*}-P_{n} g\left(\widetilde{\theta}_{n}\right)\right) \\
& +\left(\widetilde{\theta}_{n}-\theta_{n}, P_{n} g\left(\widetilde{\theta}_{n}\right)-P_{n} g\left(\theta_{n}\right)\right) \\
= & \left(\widetilde{\theta}_{n}-\theta_{n}, P_{n} g\left(\widetilde{\theta}_{n}\right)-P_{n} g\left(\theta_{n}\right)\right) \\
& +\left(\widetilde{\theta}_{n}-\theta_{n}, P_{n} g^{*}-P_{n} g(\theta)\right) \\
& +\left(\widetilde{\theta}_{n}-\theta_{n}, P_{n} g(\theta)-P_{n} g\left(\widetilde{\theta}_{n}\right)\right) \\
\leq & 2 K_{2}\left\|\widetilde{\theta}_{n}-\theta_{n}\right\|^{2}+4 K_{2}\left\|\widetilde{\theta}_{n}-\theta\right\|^{2} \\
& +\left(\widetilde{\theta}_{n}-\theta_{n}, P_{n} g^{*}-P_{n} g(\theta)\right),
\end{aligned}
$$

$$
\begin{aligned}
& \left(\widetilde{v}_{n}(s)-v_{n}(s), P_{n}\left(f^{*}(s)-\sin u_{n}(s)\right)\right) \\
& \leq\left\|\widetilde{v}_{n}-v_{n}\right\|^{2}+\frac{1}{2}\left\|\widetilde{u}_{n}-u_{n}\right\|^{2}+\frac{1}{2}\left\|\widetilde{u}_{n}-u\right\|^{2} \\
& +\left(\widetilde{v}_{n}-v_{n}, P_{n} f^{*}-P_{n} \sin u\right), \\
& \left\|P_{n} \sigma_{1}^{*}(s, z)-P_{n} \sigma_{1}\left(u_{n}\right)\right\|^{2} \\
& =\left\|P_{n} \sigma_{1}(u)-P_{n} \sigma_{1}\left(u_{n}\right)\right\|^{2}-\left\|P_{n} \sigma_{1}^{*}-P_{n} \sigma_{1}(u)\right\|^{2} \\
& +2\left(P_{n} \sigma_{1}^{*}-P_{n} \sigma_{1}(u),\right. \\
& \left.\quad P_{n} \sigma_{1}(u)-P_{n} \sigma_{1}\left(u_{n}\right)\right) .
\end{aligned}
$$

Hence, using the conditions $\mathrm{C}_{1}$ and $\mathrm{C}_{2}$ again, we get

$$
\begin{gathered}
E \int_{0}^{t} \int_{Z} \rho(s)\left\|P_{n} \sigma_{1}^{*}-P_{n} \sigma_{1}\left(u_{n}\right)\right\|^{2} \eta_{1}(d z, d s) \\
=E \int_{0}^{t} \rho(s) \int_{Z}\left\|P_{n} \sigma_{1}^{*}-P_{n} \sigma_{1}\left(u_{n}\right)\right\|^{2} \nu(d z) d s \\
=E \int_{0}^{t} \rho(s) \int_{Z}\left[\left\|P_{n} \sigma_{1}(u)-P_{n} \sigma_{1}\left(u_{n}\right)\right\|^{2}\right. \\
\left.-\left\|P_{n} \sigma_{1}^{*}-P_{n} \sigma_{1}(u)\right\|^{2}\right] v(d z) d s \\
+2 E \int_{0}^{t} \rho(s) \int_{Z}\left(P_{n} \sigma_{1}^{*}-P_{n} \sigma_{1}(u), P_{n} \sigma_{1}(u)\right. \\
\left.\quad-P_{n} \sigma_{1}\left(u_{n}\right)\right) \nu(d z) d s \\
\leq k_{2} E \int_{0}^{t} \rho(s)\left\|u-u_{n}\right\|^{2} d s \\
-E \int_{0}^{t} \rho(s)\left\|P_{n} \sigma_{1}^{*}-P_{n} \sigma_{1}(u)\right\|_{L^{2}\left(Z, v L^{2}\right)}^{2} d s \\
+2 E \int_{0}^{t} \rho(s) \int_{Z}\left(P_{n} \sigma_{1}^{*}-P_{n} \sigma_{1}(u), P_{n} \sigma_{1}(u)\right. \\
\left.\quad-P_{n} \sigma_{1}\left(u_{n}\right)\right) \nu(d z) d s,
\end{gathered}
$$

$E \int_{0}^{t} \int_{Z} \rho(s)\left\|P_{n} \sigma_{2}^{*}-P_{n} \sigma_{2}\left(\theta_{n}\right)\right\|^{2} \eta_{2}(d z, d s)$

$$
\begin{aligned}
& \leq k_{2} E \int_{0}^{t} \rho(s)\left\|\theta-\theta_{n}\right\|^{2} d s \\
& -E \int_{0}^{t} \rho(s)\left\|P_{n} \sigma_{2}^{*}-P_{n} \sigma_{2}(\theta)\right\|_{L^{2}\left(Z, v ; L^{2}\right)}^{2} d s \\
& +2 E \int_{0}^{t} \rho(s) \int_{Z}\left(P_{n} \sigma_{2}^{*}-P_{n} \sigma_{2}(\theta), P_{n} \sigma_{2}(\theta)\right. \\
& \left.-P_{n} \sigma_{2}\left(\theta_{n}\right)\right) \nu(d z) d s .
\end{aligned}
$$

Substituting (44)-(46) into (43), we have

$$
\begin{gathered}
E\left\{\rho ( t ) \left(\left\|\widetilde{v}_{n}(t)-v_{n}(t)\right\|^{2}+\left\|\tilde{u}_{n}(t)-u_{n}(t)\right\|_{1}^{2}\right.\right. \\
\left.\left.+\left\|\widetilde{\theta}_{n}(t)-\theta_{n}(t)\right\|^{2}\right)\right\}
\end{gathered}
$$




$$
\begin{aligned}
& +E \int_{0}^{t} \rho(s)\left[2 \alpha_{1}\left\|\widetilde{v}_{n}(s)-v_{n}(s)\right\|_{1}^{2}\right. \\
& \left.+\left\|\tilde{\theta}_{n}(s)-\theta_{n}(s)\right\|_{1}^{2}\right] d s \\
& +E \int_{0}^{t} \rho(s)\left\|P_{n} \sigma_{1}^{*}-P_{n} \sigma_{1}(u)\right\|_{L^{2}\left(Z, v ; L^{2}\right)}^{2} d s \\
& +E \int_{0}^{t} \rho(s)\left\|P_{n} \sigma_{2}^{*}-P_{n} \sigma_{2}(u)\right\|_{L^{2}\left(Z, v ; L^{2}\right)}^{2} d s \\
& \leq E \int_{0}^{t} \rho^{\prime}(s)\left[\left\|\widetilde{u}_{n}(s)-u_{n}(s)\right\|_{1}^{2}+\left\|\widetilde{v}_{n}(s)-v_{n}(s)\right\|^{2}\right. \\
& \left.+\left\|\widetilde{\theta}_{n}(s)-\theta_{n}(s)\right\|^{2}\right] d s \\
& +E \int_{0}^{t} \rho(s)\left[\beta \lambda\left\|\widetilde{u}_{n}(s)-u_{n}(s)\right\|_{1}^{2}+2 \beta\left\|\widetilde{v}_{n}(s)-v_{n}(s)\right\|^{2}\right. \\
& \left.+4 K_{2}\left\|\widetilde{\theta}_{n}(s)-\theta_{n}(s)\right\|^{2}\right] d s \\
& +E \int_{0}^{t} \rho(s)\left[\left\|\widetilde{u}_{n}-u\right\|^{2}+2\left(\widetilde{v}_{n}-v_{n}, P_{n} f^{*}-P_{n} \sin u\right)\right] d s \\
& +2 E \int_{0}^{t} \rho(s)\left[4 K_{2}\left\|\widetilde{\theta}_{n}-\theta\right\|^{2}\right. \\
& \left.+\left(\widetilde{\theta}_{n}-\theta_{n}, P_{n} g^{*}-P_{n} g(\theta)\right)\right] d s \\
& +2 E \int_{0}^{t} \rho(s) \int_{Z}\left(P_{n} \sigma_{1}^{*}-P_{n} \sigma_{1}(u), P_{n} \sigma_{1}(u)\right. \\
& \left.-P_{n} \sigma_{1}\left(u_{n}\right)\right) v(d z) d s \\
& +2 E \int_{0}^{t} \rho(s) \int_{Z}\left(P_{n} \sigma_{2}^{*}-P_{n} \sigma_{2}(\theta), P_{n} \sigma_{2}(\theta)\right. \\
& \left.-P_{n} \sigma_{2}\left(\theta_{n}\right)\right) v(d z) d s \\
& +k_{2} E \int_{0}^{t} \rho(s)\left[\left\|u-u_{n}\right\|^{2}+\left\|\theta-\theta_{n}\right\|^{2}\right] d s,
\end{aligned}
$$

where $\lambda$ is the Sobolev embedding constant such that $\|u(\cdot)\|^{2} \leq \lambda\|u(\cdot)\|_{1}^{2}$. Choosing $\delta=\max \left\{2 \beta, \beta \lambda, 4 K_{2}\right\}$, we get $\rho^{\prime}(s)+\delta \rho(s)=0$; hence

$$
\begin{aligned}
& E\left\{\rho ( t ) \left(\left\|\widetilde{v}_{n}(t)-v_{n}(t)\right\|^{2}+\left\|\widetilde{u}_{n}(t)-u_{n}(t)\right\|_{1}^{2}\right.\right. \\
& \left.\left.\quad+\left\|\widetilde{\theta}_{n}(t)-\theta_{n}(t)\right\|^{2}\right)\right\} \\
& +E \int_{0}^{t} \rho(s)\left[2 \alpha_{1}\left\|\widetilde{v}_{n}(s)-v_{n}(s)\right\|_{1}^{2}+\left\|\widetilde{\theta}_{n}(s)-\theta_{n}(s)\right\|_{1}^{2}\right] d s \\
& \quad+E \int_{0}^{t} \rho(s)\left\|P_{n} \sigma_{1}^{*}-P_{n} \sigma_{1}(u)\right\|_{L^{2}\left(Z, v ; L^{2}\right)}^{2} d s \\
& +E \int_{0}^{t} \rho(s)\left\|P_{n} \sigma_{2}^{*}-P_{n} \sigma_{2}(u)\right\|_{L^{2}\left(Z, \gamma ; L^{2}\right)}^{2} d s
\end{aligned}
$$

$$
\begin{gathered}
\leq E \int_{0}^{t} \rho(s)\left[\left\|\widetilde{u}_{n}-u\right\|^{2}+2\left(\widetilde{v}_{n}-v_{n}, P_{n} f^{*}-P_{n} \sin u\right)\right] d s \\
+2 E \int_{0}^{t} \rho(s)\left[4 K_{2}\left\|\widetilde{\theta}_{n}-\theta\right\|^{2}\right. \\
\left.+\left(\widetilde{\theta}_{n}-\theta_{n}, P_{n} g^{*}-P_{n} g(\theta)\right)\right] d s \\
+2 E \int_{0}^{t} \rho(s) \int_{Z}\left(P_{n} \sigma_{1}^{*}-P_{n} \sigma_{1}(u), P_{n} \sigma_{1}(u)\right. \\
+2 E \int_{0}^{t} \rho(s) \int_{Z}\left(P_{n} \sigma_{2}^{*}-P_{n} \sigma_{2}(\theta), P_{n} \sigma_{2}(\theta)\right. \\
\left.\quad-P_{n} \sigma_{2}\left(\theta_{n}\right)\right) \nu(d z) d s \\
+k_{2} E \int_{0}^{t} \rho(s)\left[\left\|u-u_{n}\right\|^{2}+\left\|\theta-\theta_{n}\right\|^{2}\right] d s .
\end{gathered}
$$

Replacing $t$ by $\tau_{N}$ in (48),

$$
\begin{aligned}
& E\left\{\rho ( \tau _ { N } ) \left(\left\|\left(\widetilde{v}_{n}-v_{n}\right)\left(\tau_{N}\right)\right\|^{2}+\left\|\left(\widetilde{u}_{n}-u_{n}\right)\left(\tau_{N}\right)\right\|_{1}^{2}\right.\right. \\
& \left.\left.+\left\|\left(\widetilde{\theta}_{n}-\theta_{n}\right)\left(\tau_{N}\right)\right\|^{2}\right)\right\} \\
& +E \int_{0}^{\tau_{N}} \rho(s)\left[2 \alpha_{1}\left\|\widetilde{v}_{n}(s)-v_{n}(s)\right\|_{1}^{2}\right. \\
& \left.+\left\|\widetilde{\theta}_{n}(s)-\theta_{n}(s)\right\|_{1}^{2}\right] d s \\
& +E \int_{0}^{\tau_{N}} \rho(s)\left\|P_{n} \sigma_{1}^{*}-P_{n} \sigma_{1}(u)\right\|_{L^{2}\left(Z, v ; L^{2}\right)}^{2} d s \\
& +E \int_{0}^{\tau_{N}} \rho(s)\left\|P_{n} \sigma_{2}^{*}-P_{n} \sigma_{2}(u)\right\|_{L^{2}\left(Z, v ; L^{2}\right)}^{2} d s \\
& \leq E \int_{0}^{\tau_{N}} \rho(s)\left[\left\|\widetilde{u}_{n}-u\right\|^{2}+2\left(\widetilde{v}_{n}-v_{n}, P_{n} f^{*}-P_{n} \sin u\right)\right] d s \\
& +2 E \int_{0}^{\tau_{N}} \rho(s)\left[4 K_{2}\left\|\widetilde{\theta}_{n}-\theta\right\|^{2}\right. \\
& +k_{2} \int_{0}^{\tau_{N}} \rho(s)\left[\left\|u-u_{n}\right\|^{2}+\left\|\theta-\theta_{n}\right\|^{2}\right] d s . \\
& +2 E \int_{0}^{\tau_{N}} \rho(s) \int_{Z}\left(P_{n} \sigma_{1}^{*}-P_{n} \sigma_{1}(u), P_{n} \sigma_{1}(u)\right. \\
& \left.+2 E \int_{0}^{\tau_{N}} \rho(s) \int_{Z}\left(P_{n} \sigma_{2}^{*}-P_{n} \sigma_{2}(\theta), P_{n} \sigma_{2}(\theta)\right)\right] d s \\
& \left.\quad-P_{n} \sigma_{1}\left(u_{n}\right)\right) v(d z) d s
\end{aligned}
$$


Since $1_{\left[0, \tau_{N}\right]} \rho(s)\left(\sigma_{1}^{*}-\sigma_{1}(u)\right) \in L^{2}\left(Z, v ; L^{2}\right)$ implies that $1_{\left[0, \tau_{N}\right]} \rho(s)\left(P_{n} \sigma_{1}^{*}-P_{n} \sigma_{1}(u)\right) \in L^{2}\left(Z, v ; L^{2}\right)$, due to (31) and Sobolev embedding theorem, we have $\left\|u_{n}-\widehat{u}\right\|_{L^{2}(0, L)} \rightarrow 0$ as $n \rightarrow \infty$; hence

$$
\begin{aligned}
& E \int_{0}^{\tau_{N}} \rho(s)\left\|P_{n} \sigma_{1}(u)-P_{n} \sigma_{1}\left(u_{n}\right)\right\|_{L^{2}\left(Z, v ; L^{2}\right)}^{2} d s \\
& \quad \leq E \int_{0}^{\tau_{N}} \rho(s)\left\|\sigma_{1}(u)-\sigma_{1}\left(u_{n}\right)\right\|_{L^{2}\left(Z, v ; L^{2}\right)}^{2} d s \\
& \quad \leq k_{2} E \int_{0}^{\tau_{N}} \rho(s)\left\|u-u_{n}\right\|_{L^{2}(0, L)}^{2} d s \\
& \quad=k_{2} E \int_{0}^{\tau_{N}} \rho(s)\left\|\widehat{u}-u_{n}\right\|_{L^{2}(0, L)}^{2} d s \longrightarrow 0 \quad(n \longrightarrow \infty) ;
\end{aligned}
$$

then we have

$$
\begin{array}{r}
\lim _{n \rightarrow \infty} E \int_{0}^{\tau_{N}} \rho(s) \int_{Z}\left(P_{n} \sigma_{1}^{*}-P_{n} \sigma_{1}(u), P_{n} \sigma_{1}(u)\right. \\
\left.-P_{n} \sigma_{1}\left(u_{n}\right)\right) \nu(d z) d s=0 .
\end{array}
$$

Similarly, we can prove that

$$
\begin{array}{r}
\lim _{n \rightarrow \infty} E \int_{0}^{\tau_{N}} \rho(s) \int_{Z}\left(P_{n} \sigma_{2}^{*}-P_{n} \sigma_{2}(\theta), P_{n} \sigma_{2}(\theta)\right. \\
\left.-P_{n} \sigma_{2}\left(\theta_{n}\right)\right) \nu(d z) d s=0 .
\end{array}
$$

Owing to (31)-(34) the sequence $P_{n} g^{*}-P_{n} g(\theta)$ is bounded in $L^{2}\left(\Omega \times[0, T] ; L^{2}\right)$, and by the Sobolev embedding theorem,

$$
\begin{aligned}
& \lim _{n \rightarrow \infty} E \int_{0}^{\tau_{N}} \rho(s)\left(\widetilde{\theta}_{n}-\theta_{n}, P_{n} g^{*}-P_{n} g(\theta)\right) d s \\
& \leq C \lim _{n \rightarrow \infty} E \int_{0}^{\tau_{N}} \rho(s)\left\|\widetilde{\theta}_{n}-\theta_{n}\right\|^{2} d s \\
& \quad=C \lim _{n \rightarrow \infty} E \int_{0}^{\tau_{N}} \rho(s)\left\|P_{n} \theta-P_{n} \theta_{n}\right\|^{2} d s \\
& \leq C \lim _{n \rightarrow \infty} E \int_{0}^{\tau_{N}} \rho(s)\left\|\theta-\theta_{n}\right\|^{2} d s \\
& \quad=C \lim _{n \rightarrow \infty} E \int_{0}^{\tau_{N}} \rho(s)\left\|\hat{\theta}-\theta_{n}\right\|^{2} d s=0 .
\end{aligned}
$$

Similarly, we can prove that

$$
\begin{gathered}
\lim _{n \rightarrow \infty} E \int_{0}^{\tau_{N}} \rho(s)\left(\widetilde{v}_{n}-v_{n}, P_{n} f^{*}-P_{n} \sin u\right) d s \\
\leq C \lim _{n \rightarrow \infty} E \int_{0}^{\tau_{N}} \rho(s)\left\|\widetilde{v}_{n}-v_{n}\right\|^{2} d s=0, \\
\lim _{n \rightarrow \infty} E \int_{0}^{\tau_{N}} \rho(s)\left[\left\|u-u_{n}\right\|^{2}+\left\|\widetilde{u}_{n}-u\right\|^{2}+\left\|\widetilde{\theta}_{n}-\theta\right\|^{2}\right. \\
\left.+\left\|\theta-\theta_{n}\right\|^{2}\right] d s=0 .
\end{gathered}
$$

Therefore, the limits (51)-(54) imply that (39) holds true.
Next, due to the property of $g$, we see that, for all $\psi \epsilon$ $L^{2}\left(\Omega \times[0, T] ; L^{2}(0, L)\right)$,

$$
\begin{aligned}
& \lim _{n \rightarrow \infty} E \int_{0}^{\tau_{N}}\left(g\left(\theta_{n}(s)\right)-g(\theta(s)), \psi(s)\right) d s \\
& \leq C \lim _{n \rightarrow \infty} E \int_{0}^{\tau_{N}}\left\|\theta_{n}(s)-\theta(s)\right\|\|\psi(s)\| d s=0, \\
& \lim _{n \rightarrow \infty} E \int_{0}^{\tau_{N}}\left(\sin u_{n}(s)-\sin u(s), \psi(s)\right) d s=0 .
\end{aligned}
$$

From this, since $P_{n} g\left(\theta_{n}\right) \rightarrow g^{*}, P_{n} \sin u_{n} \rightarrow f^{*}$ in $L^{2}(\Omega \times$ $\left.[0, T] ; L^{2}(0, L)\right)$, and (39), we have

$$
\begin{gathered}
I_{\left[0, \tau_{N}\right]}(s) \sigma_{1}^{*}(s)=I_{\left[0, \tau_{N}\right]}(s) \sigma_{1}(u(s)), \\
I_{\left[0, \tau_{N}\right]}(s) \sigma_{2}^{*}(s)=I_{\left[0, \tau_{N}\right]}(s) \sigma_{2}(\theta(s)), \\
I_{\left[0, \tau_{N}\right]}(s) g^{*}(s)=I_{\left[0, \tau_{N}\right]}(s) g(\theta(s)), \\
I_{\left[0, \tau_{N}\right]}(s) f^{*}(s)=I_{\left[0, \tau_{N}\right]}(s) \sin u(s),
\end{gathered}
$$

for almost everywhere $(\omega, t) \in \Omega \times[0, T]$.

Putting (56) into (37), we get

$$
\left(u(t), \varphi_{1}\right)=\left(u_{0}, \varphi_{1}\right)+\int_{0}^{t}\left(v(s), \varphi_{1}\right) d s,
$$

$$
\begin{aligned}
\left(v(t), \varphi_{2}\right)= & \left(v_{0}, \varphi_{2}\right) \\
& +\int_{0}^{t}\left(\alpha_{1} v_{x x}+u_{x x}+\beta \sin u+\alpha_{2} \theta, \varphi_{2}\right) d s \\
& +\int_{0}^{t} \int_{Z}\left(\sigma_{1}(u), \varphi_{2}\right) \widetilde{\eta}_{1}(d z, d s), \\
\left(\theta(t), \varphi_{3}\right)= & \left(\theta_{0}, \varphi_{3}\right) \\
& +\int_{0}^{t}\left(\theta_{x x}-\alpha_{2} v+g(\theta), \varphi_{3}\right) d s \\
& +\int_{0}^{t} \int_{Z}\left(\sigma_{2}(\theta), \varphi_{3}\right) \widetilde{\eta_{2}}(d z, d s),
\end{aligned}
$$

a.s., $\forall\left(\varphi_{1}, \varphi_{2}, \varphi_{3}\right) \in L^{2} \times H_{0}^{1} \times H_{0}^{1}, t \in[0, T]$.

From the property of $\tau_{N}$, we obtain $P\left(\bigcup_{N=1}^{\infty}\left\{\tau_{N}=T\right\}\right)=$ 1 ; let

$$
\begin{aligned}
\Omega^{\prime}:=\left\{\omega \in \Omega ; \omega \in \bigcup_{N=1}^{\infty}\left\{\tau_{N}=T\right\},\right. \\
(u, v, \theta)(\omega, t) \text { satisfies }(57)\} .
\end{aligned}
$$

and $P\left(\Omega^{\prime}\right)=1$. For $\omega \in \Omega^{\prime}$ there exists $N^{\prime}>0$ such that $\tau_{N}=T$ for all $N \geq N^{\prime}$. 
Then, $(u(t), v(t), \theta(t))$ is a solution to $(10)$.

Uniqueness. Set $\left(u_{1}, v_{1}, \theta_{1}\right)$ and $\left(u_{2}, v_{2}, \theta_{2}\right)$ as two solutions of (10); thus

$$
\begin{aligned}
&\left(\left(u_{1}-u_{2}\right)(t), \varphi_{1}\right)= \int_{0}^{t}\left(v(s), \varphi_{1}\right) d s \\
&\left(\left(v_{1}-v_{2}\right)(t), \varphi_{2}\right)= \int_{0}^{t}\left(\alpha_{1}\left(v_{1}-v_{2}\right)_{x x}+\left(u_{1}-u_{2}\right)_{x x}\right. \\
&\left.+\beta\left(\sin u_{2}-\sin u_{2}\right), \varphi_{2}\right) d s \\
&+\int_{0}^{t} \beta \alpha_{2}\left(\left(\theta_{1}-\theta_{2}\right), \varphi_{2}\right) d s \\
&+\int_{0}^{t} \int_{Z}\left(\sigma_{1}\left(u_{1}\right)-\sigma_{1}\left(u_{2}\right), \varphi_{2}\right) \\
& \times \widetilde{\eta_{1}}(d z, d s), \\
&\left(\left(\theta_{1}-\theta_{2}\right)(t), \varphi_{3}\right)= \int_{0}^{t}\left(\left(\theta_{1}-\theta_{2}\right)_{x x}-\alpha_{2}\left(v_{1}-v_{2}\right)\right. \\
&\left.+\int_{0}^{t} \int_{Z}\left(\sigma_{2}\right)-g\left(\theta_{2}\right), \varphi_{3}\right) d s \\
&\left.\times \widetilde{\eta_{2}}(d z, d s), \sigma_{2}\left(\theta_{2}\right), \varphi_{3}\right)
\end{aligned}
$$

a.s., $\forall\left(\varphi_{1}, \varphi_{2}, \varphi_{3}\right) \in L^{2} \times H_{0}^{1} \times H_{0}^{1}, t \in[0, T]$.

From a similar argument as in the proof of existence, by the B-D-G inequality, conditions $\mathrm{C}_{1}, \mathrm{C}_{2}$, and the Gronwall lemma, one can easily show that

$$
E\left(\left\|u_{1}(t)-u_{2}(t)\right\|_{1}^{2}+\left\|\theta_{1}(t)-\theta_{2}(t)\right\|_{1}^{2}\right)=0
$$

for $t \in[0, T]$; thus $P\left(u_{1}(t)=u_{2}(t), \theta_{1}(t)=\theta_{2}(t)\right)=1$ for all $t \in[0, T]$.

We complete the proof of the theorem.

\section{Asymptotic Behavior}

In this section, we briefly discuss the long time behavior of the strong solutions of the stochastic nonlinear thermoelastic system coupled sine-Gordon equation driven by jump noise.

Following the idea in [6], we assume that there exists a constant $a>0$ such that

$$
\begin{gathered}
\gamma=\max \left\{a+\beta, a+1+K_{1}+k_{1}\right\}>0, \\
\xi=\min \left\{\frac{2 \alpha_{1}}{\lambda}, \frac{2}{\lambda}\right\}>0,
\end{gathered}
$$

where $\lambda$ is defined in Section 3.

Theorem 4. Suppose that the conditions for Theorem 3 hold true. If $\xi-\gamma-a<0$, then the solution $\{(u(t), v(t), \theta(t))\}_{t \in[0, T]}$ of the problem (10) satisfies

$$
E\left(\left\|u_{t}(t)\right\|^{2}+\|\theta(t)\|^{2}\right) \leq e^{(\xi-\gamma-a) t}\left(C_{0}+C_{1} t+C_{2} t^{2}\right),
$$

where $C_{i}(i=0,1,2)$ are the positive constants.
Proof. Set $\rho(t)=e^{a t}$, using Itô's formula to the processes $\rho(t)\|v(t)\|^{2}, \quad \rho(t)\|u(t)\|_{1}^{2}$, and $\rho(t)\|\theta(t)\|^{2}$, respectively; we obtain

$$
\begin{aligned}
& \rho(t)\|v(t)\|^{2}+\rho(t)\|u(t)\|_{1}^{2} \\
& =\left\|v_{0}\right\|^{2}+\left\|u_{0}\right\|_{1}^{2}+\int_{0}^{t} \rho^{\prime}(s)\|v(s)\|^{2} d s \\
& -2 \alpha_{1} \int_{0}^{t} \rho(s)\|v(s)\|_{1}^{2} d s \\
& +\int_{0}^{t} \rho^{\prime}(s)\|u(s)\|_{1}^{2} d s \\
& +2 \beta \int_{0}^{t} \rho(s)(v(s), \sin u(s)) d s \\
& +2 \alpha_{2} \int_{0}^{t} \rho(s)(v(s), \theta(s)) d s \\
& +2 \int_{0}^{t} \int_{Z} \rho(s)\left(v(s-), \sigma_{1}(u)\right) \widetilde{\eta_{1}}(d z, d s) \\
& +\int_{0}^{t} \int_{Z} \rho(s)\left\|\sigma_{1}(u)\right\|^{2} \eta_{1}(d z, d s), \\
& \rho(t)\|\theta(t)\|^{2}=\left\|\theta_{0}\right\|^{2}-2 \int_{0}^{t} \rho(s)\|\theta(s)\|_{1}^{2} d s \\
& +\int_{0}^{t} \rho^{\prime}(s)\|\theta(s)\|^{2} d s \\
& -2 \alpha_{2} \int_{0}^{t} \rho(s)(v(s), \theta(s)) d s \\
& +2 \int_{0}^{t} \rho(s)(\theta(s), g(\theta(s))) d s \\
& +2 \int_{0}^{t} \int_{Z} \rho(s)\left(\theta(s-), \sigma_{2}(\theta)\right) \widetilde{\eta_{2}}(d z, d s) \\
& +\int_{0}^{t} \int_{Z} \rho(s)\left\|\sigma_{2}(\theta)\right\|^{2} \eta_{2}(d z, d s) .
\end{aligned}
$$

Taking the mathematical expectations in (63) yields

$$
\begin{aligned}
E \rho(t) & {\left[\|v(t)\|^{2}+\|u(t)\|_{1}^{2}+\|\theta(t)\|^{2}\right] } \\
& +2 \alpha_{1} E \int_{0}^{t} \rho(s)\|v(s)\|_{1}^{2} d s+2 E \int_{0}^{t} \rho(s)\|\theta(s)\|_{1}^{2} d s \\
= & {\left[\left\|v_{0}\right\|^{2}+\left\|u_{0}\right\|_{1}^{2}+\left\|\theta_{0}\right\|^{2}\right] } \\
& +E \int_{0}^{t} \rho^{\prime}(s)\left[\|u(s)\|_{1}^{2}+\|v(s)\|^{2}+\|\theta(s)\|^{2}\right] d s \\
& +2 \beta E \int_{0}^{t} \rho(s)(v(s), \sin u(s)) d s
\end{aligned}
$$




$$
\begin{aligned}
& +2 E \int_{0}^{t} \rho(s)(\theta(s), g(\theta(s))) d s \\
& +E \int_{0}^{t} \int_{Z} \rho(s)\left\|\sigma_{1}(u)\right\|^{2} \eta_{1}(d s, d z) \\
& +E \int_{0}^{t} \int_{Z} \rho(s)\left\|\sigma_{2}(\theta)\right\|^{2} \eta_{2}(d s, d z) .
\end{aligned}
$$

From Hölder's inequality, Young's inequality, and condition $\mathrm{C}_{1}$, we have

$$
\begin{aligned}
& 2 \beta E \int_{0}^{t} \rho(s)(v(s), \sin u(s)) d s \\
& \quad \leq \beta E \int_{0}^{t} \rho(s)\|v(s)\|^{2} d s+\beta E \int_{0}^{t} \rho(s)\|u(s)\|^{2} d s \\
& 2 E \int_{0}^{t} \rho(s)(\theta(s), g(\theta(s))) d s \\
& \quad \leq E \int_{0}^{t} \rho(s)\|\theta(s)\|^{2} d s+K_{1} E \int_{0}^{t} \rho(s)\|\theta(s)\|^{2} d s, \\
& E \int_{0}^{t} \int_{Z} \rho(s)\left\|\sigma_{1}(u)\right\|^{2} \eta_{1}(d s, d z) \\
& \quad=E \int_{0}^{t} \int_{Z} \rho(s)\left\|\sigma_{1}(u)\right\|^{2} v(d z) d s \\
& \quad \leq k_{1} E \int_{0}^{t} \rho(s)\|u\|^{2} d s, \\
& \quad \leq \sum_{0}^{t} \int_{Z} \rho(s)\left\|\sigma_{2}(\theta)\right\|^{2} \eta_{2}(d s, d z) \\
& \quad=E \int_{0}^{t} \rho(s)\|\theta\|^{2} d s . \\
& \quad \int_{Z}^{t} \rho(s)\left\|\sigma_{2}(\theta)\right\|^{2} v(d z) d s
\end{aligned}
$$

Therefore, from (64), we get

$$
\begin{aligned}
& E \rho(t)\left[\|v(t)\|^{2}+\|u(t)\|_{1}^{2}+\|\theta(t)\|^{2}\right] \\
& +2 \alpha_{1} E \int_{0}^{t} \rho(s)\|v(s)\|_{1}^{2}+2 E \int_{0}^{t} \rho(s)\|\theta(s)\|_{1}^{2} d s \\
& \leq\left[\left\|v_{0}\right\|^{2}+\left\|u_{0}\right\|_{1}^{2}+\left\|\theta_{0}\right\|^{2}\right] \\
& +a E \int_{0}^{t} \rho(s)\left[\|u(s)\|_{1}^{2}+\|v(s)\|^{2}+\|\theta(s)\|^{2}\right] d s \\
& +E \int_{0}^{t} \rho(s)\left[\beta\|v(s)\|^{2}+\left(k_{1}+\beta\right)\|u(s)\|^{2}\right. \\
& \left.+\left(1+K_{1}+k_{1}\right)\|\theta(s)\|^{2}\right] d s .
\end{aligned}
$$

Due to the embedding theorem and (61),

$$
\begin{aligned}
E \rho(t) & {\left[\|v(t)\|^{2}+\|u(t)\|_{1}^{2}+\|\theta(t)\|^{2}\right] } \\
& +\xi E \int_{0}^{t} \rho(s)\left[\|v(s)\|^{2}+\|\theta(s)\|^{2}\right] d s \\
\leq & E \rho(t)\left[\|v(t)\|^{2}+\|u(t)\|_{1}^{2}+\|\theta(t)\|^{2}\right] \\
& +\frac{2 \alpha_{1}}{\lambda} E \int_{0}^{t} \rho(s)\|v(s)\|^{2}+\frac{2}{\lambda} E \int_{0}^{t} \rho(s)\|\theta(s)\|^{2} d s \\
\leq & {\left[\left\|v_{0}\right\|^{2}+\left\|u_{0}\right\|_{1}^{2}+\left\|\theta_{0}\right\|^{2}\right] } \\
& +E \int_{0}^{t} \rho(s)\left(a+\left(k_{1}+\beta\right) \lambda\right)\|u(s)\|_{1}^{2} d s \\
& +E \int_{0}^{t} \rho(s)(a+\beta)\|v(s)\|^{2} d s \\
& +E \int_{0}^{t} \rho(s)\left(a+1+K_{1}+k_{1}\right)\|\theta(s)\|^{2} d s \\
\leq & {\left[\left\|v_{0}\right\|^{2}+\left\|u_{0}\right\|_{1}^{2}+\left\|\theta_{0}\right\|^{2}\right] } \\
& +E \int_{0}^{t} \rho(s)\left(a+\left(k_{1}+\beta\right) \lambda\right)\|u(s)\|_{1}^{2} d s \\
& +\gamma E \int_{0}^{t} \rho(s)\left[\|v(s)\|^{2}+\|\theta(s)\|^{2}\right] d s .
\end{aligned}
$$

Hence, by Theorem 3, we have

$$
\begin{aligned}
& E \rho(t)\left[\|v(t)\|^{2}+\|\theta(t)\|^{2}\right]+(\xi-\gamma) E \\
& \quad \times \int_{0}^{t} \rho(s)\left[\|v(s)\|^{2}+\|\theta(s)\|^{2}\right] d s \\
& \leq\left[\left\|v_{0}\right\|^{2}+\left\|u_{0}\right\|_{1}^{2}+\left\|\theta_{0}\right\|^{2}\right]+C t=C_{1}+C_{2} t ;
\end{aligned}
$$

Gronwall's inequality leads to

$$
\begin{aligned}
& E \rho(t)\left[\|v(t)\|^{2}+\|\theta(t)\|^{2}\right] \\
& \quad \leq e^{(\xi-\gamma) t}\left(\left\|v_{0}\right\|^{2}+\left\|\theta_{0}\right\|^{2}+C_{1} t+C_{2} t^{2}\right) .
\end{aligned}
$$

With the choice of $a, \xi, \gamma$, the assumption of Theorem 3 holds true; we obtain that

$$
E\left[\|v(t)\|^{2}+\|\theta(t)\|^{2}\right] \leq e^{(\xi-\gamma-a) t}\left(C_{0}+C_{1} t+C_{2} t^{2}\right) .
$$

This completes the proof of the theorem.

Remark 5. Since $u(x, t, \omega)$ denotes the displacement at point $(x, t)$ on an orbit $\omega \in \Omega$, a.s., $\partial u / \partial t=v$ means the velocity; the result in the Theorem 4 exhibits that the velocity is exponentially decay in time $t$ in the sense of mean square; in the view of physics, one can obtain that the displacement $u$ will tend to a constant in the large time in the sense of mean square. 


\section{Conflict of Interests}

The authors declare that there is no conflict of interests regarding the publication of this paper.

\section{Acknowledgments}

The authors are grateful to the anonymous referees for helpful comments and suggestions that greatly improved the presentation of this paper. This work is supported by NSF of China (11272277 and 11226188) and FRF for the Central Universities of China (2013ZZGH027).

\section{References}

[1] D. Applebaum, Lévy Processes and Stochastic Calculus, vol. 116, Cambridge University Press, Cambridge, UK, 2nd edition, 2009.

[2] Z. Brzeźniak, E. Hausenblas, and J. Zhu, "2D stochastic NavierStokes equations driven by jump noise," Nonlinear Analysis, vol. 79, pp. 122-139, 2013.

[3] J. Liu, L. Yan, and Y. Cang, "On a jump-type stochastic fractional partial differential equation with fractional noises," Nonlinear Analysis, vol. 75, no. 16, pp. 6060-6070, 2012.

[4] P. A. Razafimandimby and M. Sango, "Strong solution for a stochastic model of two-dimensional second grade fluids: existence, uniqueness and asymptotic behavior," Nonlinear Analysis, vol. 75, no. 11, pp. 4251-4270, 2012.

[5] R. Chiba and Y. Sugano, "Stochastic thermoelastic problem of a functionally graded plate under random temperature load," Archive of Applied Mechanics, vol. 77, pp. 215-227, 2007.

[6] P.-L. Chow, Stochastic Partial Differential Equations, Chapman \& Hall/CRC, Boca Raton, Fla, USA, 2007.

[7] G. Da Prato and J. Zabczyk, Stochastic Equations in Infinite Dimensions, Cambridge University Press, Cambridge, UK, 1996.

[8] T. Caraballo, I. Chueshov, and J. A. Langa, "Existence of invariant manifolds for coupled parabolic and hyperbolic stochastic partial differential equations," Nonlinearity, vol. 18, no. 2, pp. 747-767, 2005.

[9] P. W. Bates, K. Lu, and B. Wang, "Random attractors for stochastic reaction-diffusion equations on unbounded domains," Journal of Differential Equations, vol. 246, no. 2, pp. 845-869, 2009.

[10] G. Wang and Y. B. Tang, " $\left(L^{2}, H^{1}\right)$ random attractors for stochastic reaction-diffusion equation on unbounded domains," Abstract and Applied Analysis, vol. 2013, Article ID 279509, 23 pages, 2013.

[11] G. Wang and Y. B. Tang, "Fractal dimension of a random invariant set and applications," Journal of Applied Mathematics, vol. 2013, Article ID 415764, 5 pages, 2013.

[12] X. Fan, "Random attractor for a damped sine-Gordon equation with white noise," Pacific Journal of Mathematics, vol. 216, no. 1, pp. 63-76, 2004.

[13] E. A. Coayla-Teran, "Strong solutions for stochastic nonlinear nonlocal parabolic equations," Stochastics and Dynamics, vol. 10, no. 4, pp. 497-508, 2010.

[14] J. W. Zhang, Y. H. Ren, R. H. Wu, and T. Feng, "The global attractor of nonlinear thermoelastic coupled sine-Gordon system," Acta Physica Sinica, vol. 61, no. 11, Article ID 110404, 2012 (Chinese).
[15] H. Gao and J. E. Muñoz Rivera, "Global existence and decay for the semilinear thermoelastic contact problem," Journal of Differential Equations, vol. 186, no. 1, pp. 52-68, 2002.

[16] J. E. M. Rivera, "Energy decay rates in linear thermoelasticity," Funkcialaj Ekvacioj, vol. 35, no. 1, pp. 19-30, 1992.

[17] H. Gao, "Global attractor for the semilinear thermoelastic problem," Mathematical Methods in the Applied Sciences, vol. 26, no. 15, pp. 1255-1271, 2003. 


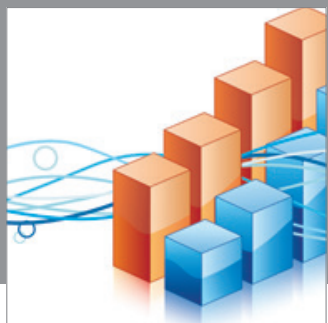

Advances in

Operations Research

mansans

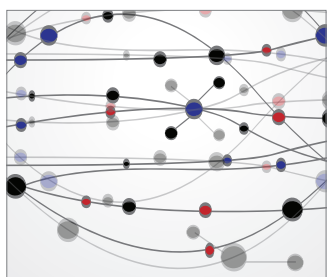

The Scientific World Journal
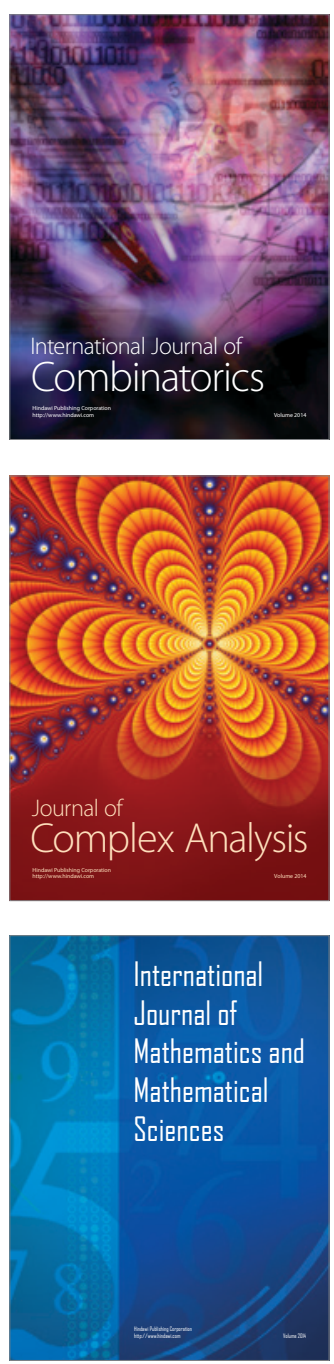
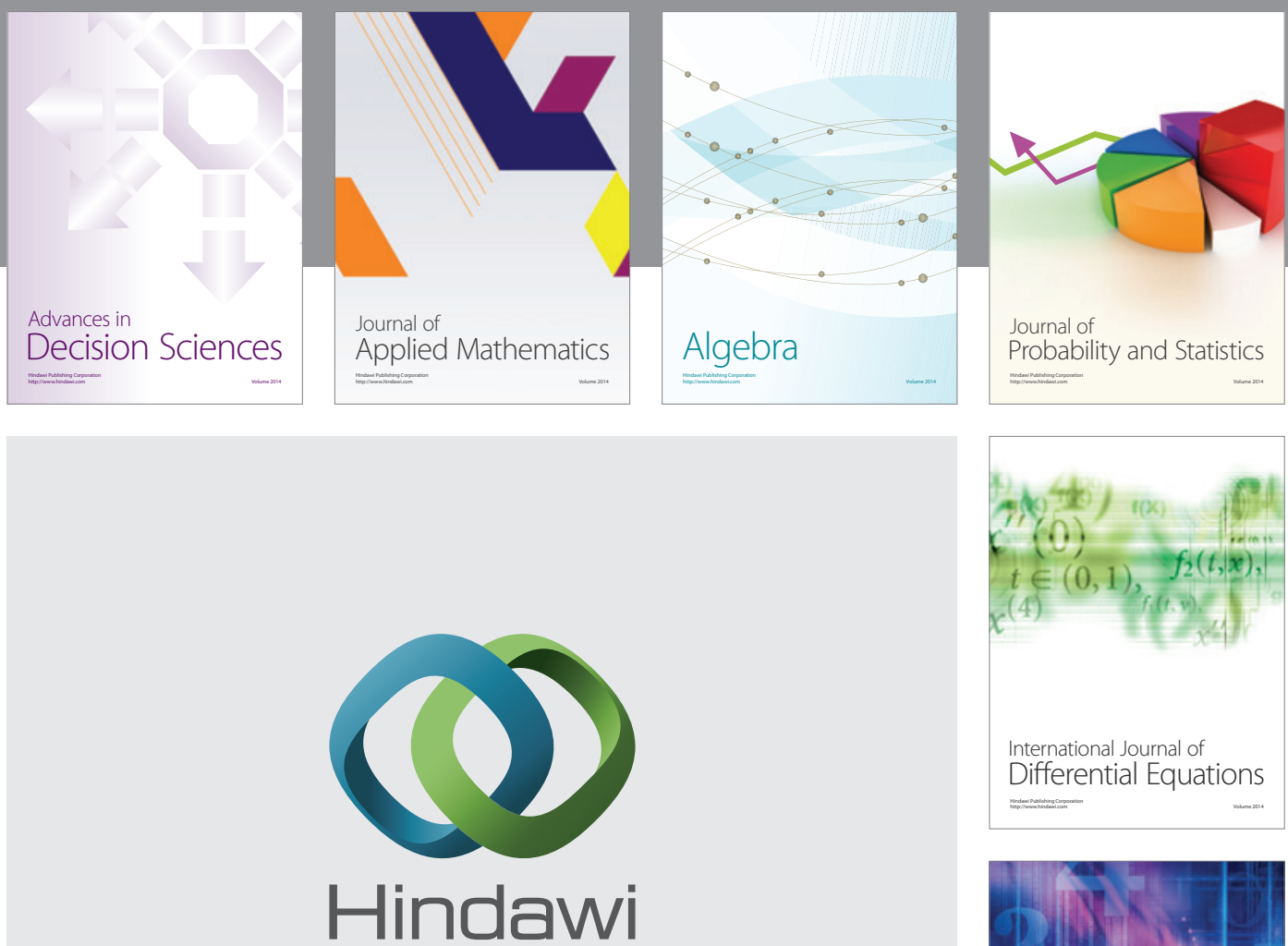

Submit your manuscripts at http://www.hindawi.com
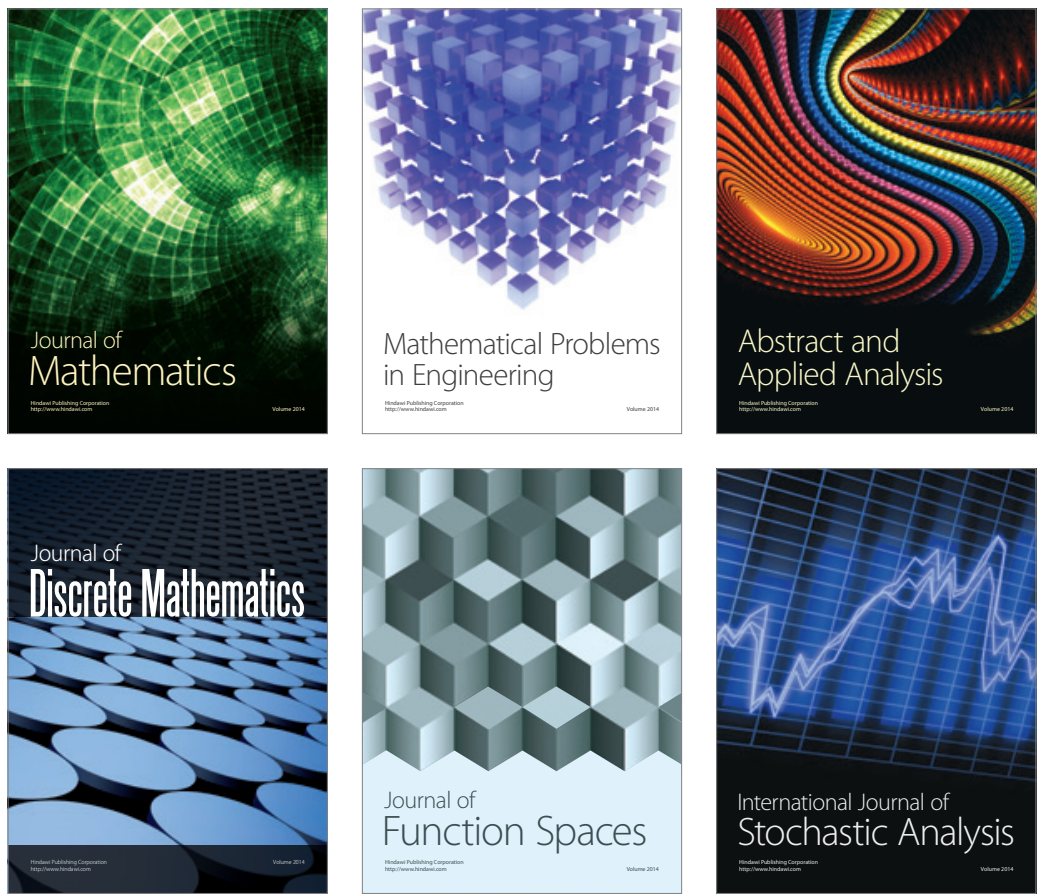

Journal of

Function Spaces

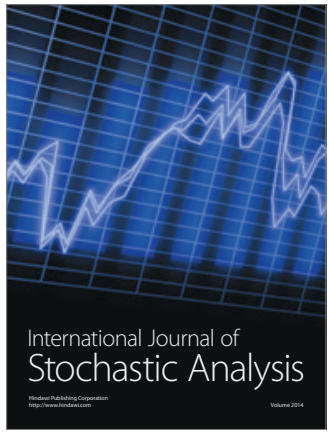

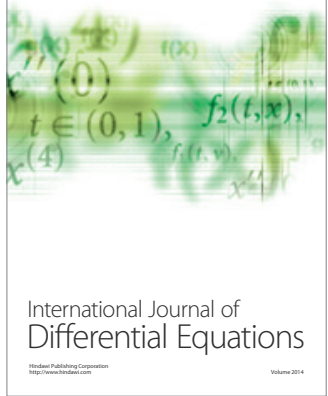
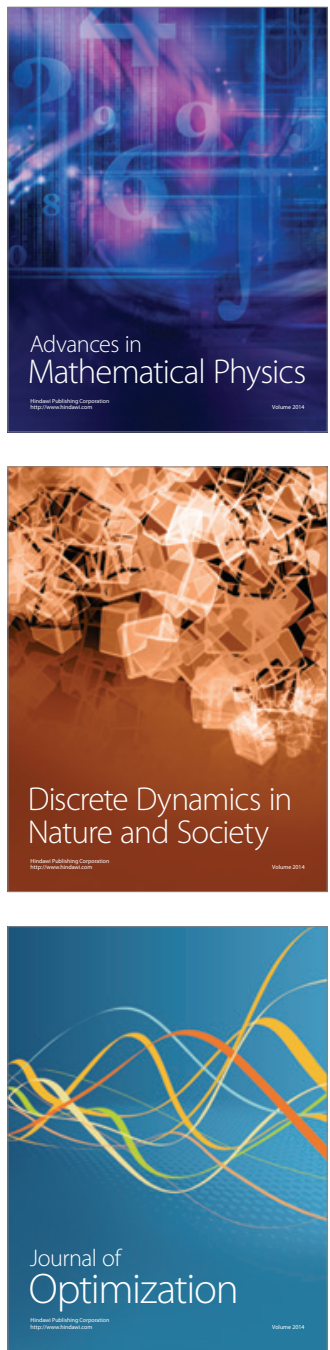